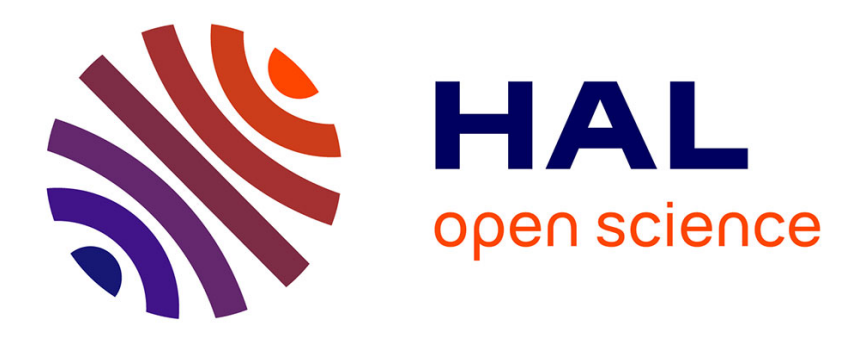

\title{
Adiabatic evolution for systems with infinitely many eigenvalue crossings
}

\author{
Alain Joye, F. Monti, S. Guerin, H. R. Jauslin
}

\section{To cite this version:}

Alain Joye, F. Monti, S. Guerin, H. R. Jauslin. Adiabatic evolution for systems with infinitely many eigenvalue crossings. Journal of Mathematical Physics, 1999, 40 (11), pp.5456-5472. 10.1063/1.533039 . hal-01233203

\section{HAL Id: hal-01233203 https://hal.science/hal-01233203}

Submitted on 24 Nov 2015

HAL is a multi-disciplinary open access archive for the deposit and dissemination of scientific research documents, whether they are published or not. The documents may come from teaching and research institutions in France or abroad, or from public or private research centers.
L'archive ouverte pluridisciplinaire HAL, est destinée au dépôt et à la diffusion de documents scientifiques de niveau recherche, publiés ou non, émanant des établissements d'enseignement et de recherche français ou étrangers, des laboratoires publics ou privés. 


\title{
Adiabatic evolution for systems with infinitely many eigenvalue crossings
}

\author{
A. Joye \\ Institut Fourier, Unité Mixte de Recherche CNRS-UJF 5582, Université de Grenoble I, \\ BP 74, 38402 Saint Martin d'Hères Cedex, France \\ F. Monti \\ Laboratoire de Physique, CNRS, Université de Bourgogne, BP 400, 21011 Dijon, France \\ and Ecole Polytechnique Fédérale de Lausanne, 1015 Lausanne, Switzerland \\ S. Guérin \\ Laboratoire de Physique, CNRS, Université de Bourgogne, BP 400, 21011 Dijon, France \\ H. R. Jauslin \\ Laboratoire de Physique, CNRS, Université de Bourgogne, BP 400, 21011 Dijon, France
}

(Received 31 December 1998; accepted for publication 22 July 1999)

We formulate an adiabatic theorem adapted to models that present an instantaneous eigenvalue experiencing an infinite number of crossings with the rest of the spectrum. We give an upper bound on the leading correction terms with respect to the adiabatic limit. The result requires only differentiability of the considered projector, and some geometric hypothesis on the local behavior of the eigenvalues at the crossings. (C) 1999 American Institute of Physics. [S0022-2488(99)00511-3]

\section{INTRODUCTION}

The availability of intense pulsed laser sources has opened a large field of possibilities to control atomic and molecular dynamical processes. One of the main theoretical tools to analyze these processes is adiabatic Floquet theory ${ }^{1}$ and references therein. The general setup can be described as follows. One considers a molecule described by a Hamiltonian $H_{0}$ acting on a Hilbert space $\mathcal{H}$, in interaction with one radiation mode of frequency $\omega$. (The description of the interaction with several modes of different frequencies can be formulated along similar lines.) Since the intensity of the field is quite large, the field is treated as a classical field. The Hamiltonian of the molecule perturbed by the electromagnetic field can be written, for example, as

$$
H=H_{0}+E M F\left(\omega t+\theta_{0}\right)
$$

where $M$ is the dipole moment operator of the molecule, $E \in \mathrm{R}$ is a parameter representing the amplitude of the radiation field, $F$ is a real valued $2 \pi$-periodic function and $\theta_{0}$ the initial phase. We assume that $H_{0}$ has a discrete spectrum. In order to describe a laser pulse the amplitude is taken as a slowly varying time dependent function $E(\epsilon t)$, where one takes, e.g., $\epsilon=1 / T_{p}$ with $T_{p}$ the duration of the pulse. A new technique that provides an efficient method for complete transfer of population is based on frequency chirping: within the pulse duration the frequency is also slowly modulated $\omega=\omega(\epsilon t)$.

This model has thus two kinds of time dependencies in the Hamiltonian: one that is periodic and another one that is slowly varying. The periodic part can be treated by Floquet methods, and the slowly varying part by adiabatic theory. Adiabatic Floquet theory is based on the following statement: Assume that in the Hamiltonian (1) the parameter $E$ and the frequency $\omega$ are made time dependent, $E(t), \omega(t)$, and $M$ stays time independent. Consider the propagator $U\left(t, t_{0} ; \theta_{0}\right)$, solution of the Schrödinger equation 


$$
i \frac{\partial}{\partial t} U\left(t, t_{0} ; \theta_{0}\right)=H\left(\omega t+\theta_{0}\right) U\left(t, t_{0} ; \theta_{0}\right), \quad U\left(t, t ; \theta_{0}\right)=1
$$

acting on the Hilbert space $\mathcal{H}$. We consider an enlarged Hilbert space by tensoring $\mathcal{H}$ with the space of square integrable functions on the unit circle: $L^{2}\left(\mathrm{~S}^{1}, \mathcal{H}\right)$. The operator $U\left(t, t_{0} ; \theta\right)$ can be lifted into the enlarged space, interpreting the $\theta$-dependence as a multiplication operator. We can then define

$$
U_{K}\left(t, t_{0}\right)=e^{-t \omega(t) \partial} U\left(t, t_{0} ; \theta\right) e^{t_{0} \omega\left(t_{0}\right) \partial}
$$

where $\partial=\partial / \partial \theta$. The statement is that Eq. (2) is equivalent to

$$
i \frac{\partial}{\partial t} U_{K}\left(t, t_{0}\right)=K(t) U_{K}\left(t, t_{0}\right)
$$

with

$$
K(t)=-i \varpi(t) \frac{\partial}{\partial \theta}+H_{0}+E(t) M F(\theta)
$$

and $\varpi(t)$ denotes an effective instantaneous frequency defined by $\varpi(t)=\omega(t)+t d \omega(t) / d t$. Assuming that the time dependence of $E(t), \omega(t)$ is slow one can develop adiabatic techniques for the evolution of (3). When $K$ has pure point spectrum, the first ingredients are the instantaneous eigenvalues and eigenvectors. They always can be written and labeled in the form

$$
\begin{gathered}
\lambda_{j, k}=\lambda_{j, 0}+k \varpi, \quad k \in \mathbb{Z} \\
\psi_{j, k}(\theta)=\psi_{j, 0}(\theta) e^{i k \theta} .
\end{gathered}
$$

The index $j$ has the same cardinality as the dimension of the Hilbert space $\mathcal{H}$. Thus, even if we take simple models with finite dimensional $\mathcal{H}$, the Floquet spectrum has infinitely many eigenvalues. As functions of $E$ and $\varpi$, these eigenvalues may exhibit crossings, which the adiabatic approximation can accommodate in case there is a finite number of them, see Refs. 2 and 3. The structure (4) of the eigenvalues is such that if we consider a slowly varying effective frequency $\varpi(t)$ that goes through 0 at some time $t_{0}$, the nature of the spectrum becomes quite different. One can encounter situations in which a branch of instantaneous eigenvalues undergoes an infinite number of crossings with other branches, or the spectrum may become suddenly continuous. Hence it becomes necessary to investigate the validity of the adiabatic theorem in such situations. Let us stress that a strictly positive time dependent frequency $\omega(t)$ may give rise quite naturally to an effective frequency $\varpi(t)$ that goes to zero. ${ }^{4}$ Indeed, consider a linear variation of $\omega$ of the form

$$
\omega(t)=\omega_{0}-a t,
$$

with $\omega_{0}, a>0$ on the time interval $\left[0, \omega_{0} / a\right)$, which is far from exotic. Then

$$
\varpi(t)=\omega_{0}-2 a t
$$

goes through zero at $t_{0}=\omega_{0} /(2 a) \in\left[0, \omega_{0} / a\right)$. As it has been shown in Refs. 4 and 5 , the possibility to vary the frequency is a powerful method to enhance the control of molecular processes driven by laser.

We will confine ourselves to the case where a branch of eigenvalue undergoes an infinity of crossings with other branches. As this situation is not generic, as actual crossings are more the exception than the rule, we give below a whole class of systems for which this situation is true. Moreover, it is probably the only case in which we get enough regularity to prove an adiabatic 
theorem. Note also that in case $\varpi(t)$ passes through 0 , the domain of $K(t)$ becomes time dependent, so that technical issues regarding regularity of the evolution operator have to be addressed. This is done in the Appendix A.

The goal of the present paper is to formulate an adiabatic theorem that can be applied to such situations with an estimate on the corrections to the adiabatic limit. Adiabatic Theorems without gap conditions are known to be true, see Ref. 6, however, in general, no estimates on the error terms are available.

While this work was motivated by the physical situation described above and discussed below in the examples, our analysis of the adiabatic approximation is model independent and can be applied to more general situations.

\section{ADIABATIC THEOREM}

\section{A. Context}

The adiabatic approximation in quantum mechanics has a long history which we will not attempt to retrace here. We refer the reader to the recent surveys ${ }^{7,8}$ and references therein. Let us simply recall here that the works following that of Born and Fock ${ }^{2}$ by Kato,${ }^{9}$ Nenciu,${ }^{10}$ and Avron, Seiler, and $\mathrm{Yaffe}^{11}$ have led to a formulation of the adiabatic theorem under the usual gap assumption that is general and where the error term is well controlled and of order $\epsilon$. In case the gap assumption is modified, the situation is less explicit. In this section, we switch back to the notation $H(\epsilon t)$ for the slowly varying time-dependent Hamiltonian. Assume $H(s)$ is smooth in $s \in[0,1]$ and there exists a spectral projector $P(s)$ of $H(s)$ which is strongly $C^{2}$ on [0,1]. Avron and Elgart have shown in Ref. 6 that the adiabatic theorem holds under these conditions, provided $P(s)$ is of finite rank, independently of any spectral considerations. A similar result was proven by Bornemann ${ }^{18}$ for discrete hamiltonians in case the set of eigenvalue crossings is of measure zero in time. The limitation of these approaches is that, in general, no estimate can be made on the rate at which the adiabatic regime is attained. In certain specific situations, an estimate on this rate is available. In the case where the spectral measure $\mu_{\varphi}$ is $\alpha$-Hölder continuous, with $\varphi$ $=P^{\prime}(s) \psi(s), \psi$ such that $P=|\psi\rangle\langle\psi|$, the rate of convergence was shown in Ref. 6 to be of order $\epsilon^{\alpha /(2+\alpha)}$. A case where the spectrum of $H(s)$ is assumed to be dense pure point is dealt with in Ref. 12. Another situation, considered in Ref. 13, where the gap hypothesis is not necessarily fulfilled occurs when $H(s)=H_{0}(s)+\epsilon H_{1}(s)$, where the domain of $H_{1}(s)$ is smaller than that of $H_{0}(s)$. In both cases, the error term remains of order $\epsilon$. In the present article, we consider another situation in which the usual gap assumption is modified and the error made in the adiabatic approximation can be estimated. We make the hypothesis that the projector $P(s)$ is associated with an eigenvalue $\lambda(s)$, in the sense that $H(s) P(s)=\lambda(s) P(s)$, for all $s \in[0,1]$. We assume that $\lambda(s)$ is isolated in the spectrum except at a series of times $\left\{o_{k}\right\}_{k \in \mathbb{N}}$ accumulating at $a \in(0,1)$ where it experiences crossings with the rest of the spectrum. Requiring some conditions on the local behavior of the gap between $\lambda(s)$ and the rest of the spectrum near the crossing points $o_{k}$, we estimate the error term in the theorem without a priori knowledge on the nature of the rest of the spectrum. Note that for $s=o_{k}$ such that $\lambda\left(o_{k}\right)$ is not isolated in the spectrum, $P\left(o_{k}\right)$ does not represent the entire spectral projector associated with the eigenvalue $\lambda\left(o_{k}\right)$.

\section{B. One crossing}

Let us make more precise the regularity hypotheses under which we shall work. In order to deal with the application described above, we will assume the Hamiltonian is unbounded. This causes technical difficulties motivating the part (ii) of the hypothesis below which justifies our manipulations. We show in the appendix that this assumption is verified for our models. In case $H(s)$ is bounded, this part of the assumption is automatically verified.

(H0) (i) We assume that for all $s \in[0,1] \backslash\{a\}, H(s)$ is a strongly $C^{1}$ self-adjoint operator defined on a dense domain $\mathcal{D}$ independent of $s$ in a separable Hilbert space $\mathcal{K}$, where $0<a<1$. 
Whereas $H(a)$ is bounded self-adjoint on $\mathcal{K}$. We also assume the existence of a projector $P(s)$ of $H(s)$ which is strongly $C^{2}$ on $[0,1]$ and such that $H(s) P(s)=P(s) H(s)=\lambda(s) P(s)$, for all $s$ $\in[0,1]$.

(ii) Further assume that the unitary evolution operators $U(s)=U(s, 0)$ and $A(s)=A(s, 0)$ generated by $H(s)$, respectively $H(s)+\epsilon i\left[P^{\prime}(s), P(s)\right]$ (see (5), (6)) are well defined for all $s$ $\in[0,1]$ and possess the properties (i) to (v) listed in Theorem A.1. Note that $P(s)$ needs not be finite dimensional and $\lambda$ is continuous.

We start by considering one crossing of $\lambda$ with the rest of the spectrum by revisiting the strategy proposed in Ref. 2, making use of the general analysis presented in Ref. 11.

Let $g(s)$ be the gap between $\lambda(s)$ and the rest of the spectrum of $H(s): g(s)$ $=\operatorname{dist}(\lambda(s), \sigma(s) \backslash\{\lambda(s)\}) \geqslant 0, s \in[0,1]$. We also introduce the bounded, strongly $C^{1}$ operator $L(s)=i\left[P^{\prime}(s), P(s)\right]$. We assume that $g^{-1}\{0\}=\{o\}$ and consider the strong differential equations on $\mathcal{D}$

$$
\begin{gathered}
i \epsilon U^{\prime}(s)=H(s) U(s), \quad U(0)=1, \\
i \epsilon A^{\prime}(s)=(H(s)+\epsilon L(s)) A(s), \quad A(0)=1 .
\end{gathered}
$$

The unitary $A$ is the so called adiabatic evolution which possesses the well known intertwining relation $A(s) P(0)=P(s) A(s){ }^{9,14}$ Finally, let $W(s)$ be defined by $W(s)=A^{-1}(s) U(s)$. We have on $\mathcal{D}$

$$
i W^{\prime}(s)=-A^{-1}(s) L(s) A(s) W(s), \quad W(0)=1,
$$

in the strong sense. To compare the adiabatic and actual evolutions, we need to compute the size of the difference of the unitary $W(s)$ at two times surrounding the crossing. This is the aim of the next result.

Lemma 2.1: Under the above assumptions, we have for any $0 \leqslant u_{0} \leqslant t<o<s \leqslant u_{1} \leqslant 1$,

$$
\left\|W\left(u_{0}\right)-W\left(u_{1}\right)\right\| \leqslant C\left(\epsilon\left|u_{0}-t\right| / g_{t}^{2}+\epsilon\left|u_{1}-s\right| / g_{s}^{2}+\epsilon / g_{t}+\epsilon / g_{s}+|s-t|\right)
$$

where $g_{t}=\inf _{u \in\left[u_{0}, t\right]} g(u), g_{s}=\inf _{u \in\left[s, u_{1}\right] g(u)}$ and the constant $C$ is uniform in $u_{0}, u_{1}, s$, and $t$ (see Fig. 1).

Remark: On the basis of the classical paper by Born and Fock, ${ }^{2}$ and the detailed analysis of crossings by Hagedorn, ${ }^{3}$ one would expect the corresponding estimate without the first two terms.

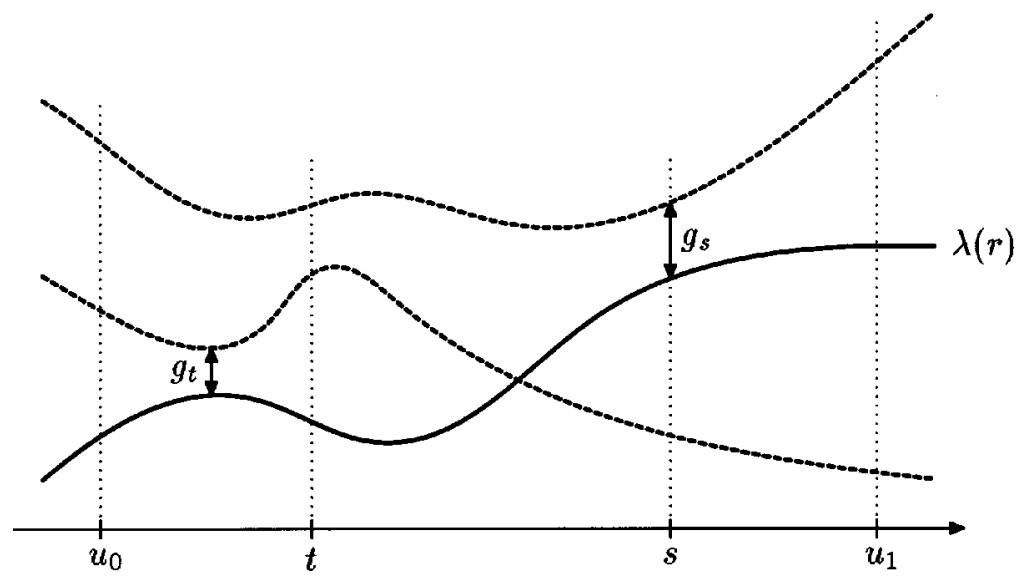

FIG. 1. The various quantities defined in Lemma 2.1. 
However, such an estimate requires more detailed knowledge of the structure of spectrum, e.g., that the gap is given by the distance between two eigenvalues, than what we assume in our general setting.

The proof of Lemma 2.1 is presented in Appendix B. The idea of the proof is to integrate Eq. (7) over the interval $\left[u_{0}, u_{1}\right]$ and then to get estimates of the sizes on each subintervals $\left[u_{0}, t\right]$, $[t, s]$, and $\left[s, u_{1}\right]$ which involves only the gaps.

Lemma 2.1 can be used to treat two standard situations:

(1) If there is a gap $G$ between $\lambda(s)$ and the rest of the spectrum, this lemma implies that the adiabatic approximation holds with an error term bounded by $C \epsilon / G^{2}$.

(2) If one starts the evolution on a crossing point which splits like $s^{\alpha}$ near 0 , we can use this lemma to show that the adiabatic approximation is valid with an error bounded by

$$
\|U(1)-A(1)\| \leqslant C \epsilon^{1 /(1+2 \alpha)}
$$

if $\epsilon$ is small enough. This is precisely the situation encountered at the beginning of the interaction of a laser pulse with frequency that is in resonance with the difference between two energy levels of the molecule. ${ }^{15,16}$

To get this estimate, we can consider only half of the problem by letting aside all the terms containing a $t$ and setting $u_{1}=1$ :

$$
\|W(1)-W(0)\| \leqslant C\left(\epsilon|1-s| / g_{s}^{2}+\epsilon / g_{s}+s\right) .
$$

This is indeed fully justified by the proof of the lemma (see Appendix B). Next, we have by hypothesis that $g(s) \geqslant g_{s}=G s^{\alpha}$ if $s$ is small. Introducing this behavior in Eq. (9), we obtain $\|W(1)-W(0)\| \leqslant C\left(\epsilon / s^{2 \alpha}+s\right)$. The result follows now by balancing the two contributions by choosing $s=s(\epsilon)=\epsilon^{1 /(1+2 \alpha)}$. Again, with more information on the spectrum, as in Refs. 2 and 3, one should be able to improve the above estimate to order $\epsilon^{1 /(1+\alpha)}$.

\section{Infinite number of crossings}

We now have all the information required to proceed to the case of an infinite number of crossings. We make the following hypotheses describing what happens in the neighborhood of each crossing (see Fig. 2).

Spectral hypotheses: There exist two partitions $\left\{u_{k}^{ \pm}\right\}_{k \in \mathbb{N}}$ of $[0, a)$ and $(a, 1]$ respectively:

$$
0=u_{0}^{-}<\cdots<u_{k-1}^{-}<u_{k}^{-} \cdots \rightarrow u_{\infty}^{-}=a=u_{\infty}^{+} \leftarrow \cdots u_{k}^{+}<u_{k-1}^{+}<\cdots<u_{0}^{+}=1
$$

such that for each $k \in \mathbb{N}^{*}$,

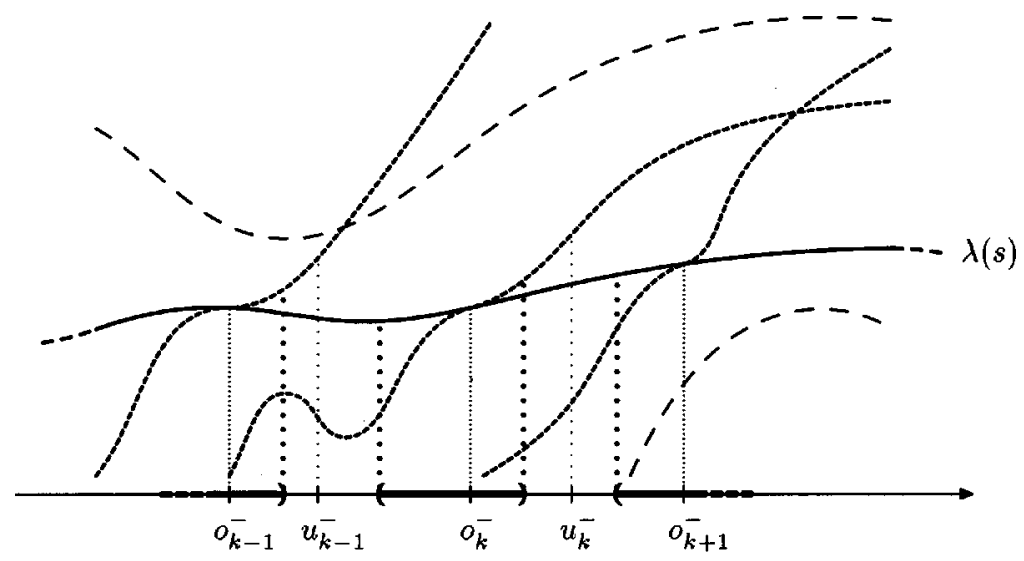

FIG. 2. Illustration of the spectral hypotheses $H 1-H 2$ on the interval $(0, a)$. The intervals $V_{l}^{-}$are represented by $(-)$. 
(H1) one can find non empty open intervals $V_{k}^{ \pm}$, which satisfy $V_{k}^{-} \subset\left[u_{k-1}^{-}, u_{k}^{-}\right]$, $V_{k}^{+} \subset\left[u_{k}^{+}, u_{k-1}^{+}\right]$and

$$
\sup _{s \in V_{k}^{ \pm}} g(s) \leqslant \inf _{t \in I_{k}^{ \pm}} g(t),
$$

where $I_{k}^{-}=\left[u_{k-1}^{-}, u_{k}^{-}\right] \backslash V_{k}^{-}$and $I_{k}^{+}=\left[u_{k}^{+}, u_{k-1}^{+}\right] \backslash V_{k}^{+}$.

$(H 2)$ there are constants $G_{ \pm}(k)>0$ and a $k$-independent positive constant $\alpha$ such that for all $s \in V_{k}^{ \pm}:$

$$
G_{ \pm}(k)\left|s-o_{k}^{ \pm}\right|^{\alpha} \leqslant g(s),
$$

for some points $o_{k}^{ \pm} \in V_{k}^{ \pm}$.

Comments: (1) These Spectral Hypotheses mean that the crossings are well separated and that they behave as power of order at most $\alpha$. Hypothesis $(H 1)$ tells us that outside the crossing regions $\left(V_{k}^{ \pm}\right)$the gaps are relatively "large." This means that the only accumulation point of small gaps is $a$.

(2) The choice of a constant exponent $\alpha$ is not as restrictive as it might look at first. Indeed, we are interested in an upper bound, so it is the greatest $\alpha$ that will determine the global behavior.

(3) In the applications, we will consider examples where $g^{-1}\{0\}=\left\{o_{k}^{ \pm}\right\}$: the set of crossing points of $\lambda(s)$ with the rest of the spectrum. This implies $\alpha>0$. But, the case of an infinite number of avoided crossings can be treated by taking $\alpha=0$ in Hypothesis $(H 2)$.

To obtain an estimate for the difference between the real evolution $U(1)$ and the adiabatic one $A(1)$, the idea is to apply Lemma 2.1 on a finite number of crossings and to take a simple integral bound [as in (B2)] over the rest of the interval surrounding $a$. The choice of the number of crossings will be optimized with respect to $\epsilon$ in order to get a simple form for the bound of the remainder term. To state the corresponding result, we need to introduce some notations. Let $\Delta_{ \pm}(k)=\max \left\{\left|u_{k}^{ \pm}-o_{k}^{ \pm}\right|,\left|u_{k-1}^{ \pm}-o_{k}^{ \pm}\right|\right\}$and $\tau_{ \pm}(k)=\max \left\{\Delta_{ \pm}(k) / G_{ \pm}^{2}(k), \Delta_{ \pm}^{\alpha}(k) / G_{ \pm}(k)\right\}$. The functions $K \mapsto\left|u_{K}^{ \pm}-a\right| / \Sigma_{k=1}^{K} \tau_{ \pm}(k)^{1 /(1+2 \alpha)}$ are monotonically decreasing to zero, so, if $\epsilon$ is small enough, we define $K_{ \pm}(\epsilon) \in \mathbb{N}^{*}$ as the greatest integer satisfying

$$
\frac{\left|u_{K}^{ \pm}-a\right|}{\sum_{k=1}^{K} \tau_{ \pm}(k)^{1 /(1+2 \alpha)}} \geqslant \epsilon^{1 /(1+2 \alpha)}
$$

This integer always exists if $\epsilon$ is sufficiently small and, by construction, $K_{ \pm}(\epsilon) \rightarrow \infty$ as $\epsilon \rightarrow 0$.

Theorem 2.1: For $\epsilon$ small enough, under (H0) and the spectral hypotheses $(H 1),(H 2)$ and provided that

$$
\boldsymbol{\varsigma}\left(\epsilon \tau_{ \pm}(k)\right)^{1 /(1+2 \alpha)} \leqslant\left|V_{k}^{ \pm}\right| / 2 \text { for all } 1 \leqslant k \leqslant K_{ \pm}(\epsilon) \text {, }
$$

for some constant $\varsigma>0$, we have that

$$
U(1)=A(1)+O\left(\max \left\{\left|u_{K_{-}(\epsilon)}^{-}-a\right|,\left|u_{K_{+}(\epsilon)}^{+}-a\right|\right\}\right) .
$$

Hence, as $\lim _{\epsilon \rightarrow 0} K_{ \pm}(\epsilon)=\infty, \quad\|U(1)-A(1)\|$ goes to zero for $\epsilon \rightarrow 0$ as fast as $\max \left\{\left|u_{K_{-}(\epsilon)}^{-}-a\right|,\left|u_{K_{+}(\epsilon)}^{+}-a\right|\right\}$.

Remarks: (1) The theorem states that the error can be estimated provided we can compute the critical value $K_{ \pm}(\epsilon)$. Further considerations on the practical aspects of this computation are given in the next section.

(2) Condition (13) implies that the size of the intervals $V_{k}^{ \pm}$cannot be too small with respect to $\epsilon \tau_{ \pm}(k)$. 
(3) While we shall apply the theorem in a situation where the spectrum is simple and pure point, the theorem remains valid under the sole existence of an eigenvalue separated from the rest of the spectrum by gaps with the properties stated in $(H 1)-(H 2)$, without any knowledge on the rest of the spectrum or restriction on the dimension of $P(s)$.

(4) The introduction of an adjustable constant $s$ is necessary in the following application to satisfy the hypothesis of the theorem.

\section{APPLICATION}

We can obtain more explicit estimates on the rest by considering some specific behavior at the crossings.

Let us introduce the following notation: $F_{k} \sim f(k)$ means that there exist two constants $0<c_{1}<c_{2}<\infty$ such that $c_{1} f(k) \leqslant F_{k} \leqslant c_{2} f(k)$ for $k \in \mathbb{N}^{*}$ large enough. We have the

Proposition 3.1: Assume the hypothesis of Theorem 2.1 and the following behavior for the relevant quantities:

$$
\begin{gathered}
\left|u_{k}^{ \pm}-a\right|=C_{1} / k^{\beta}+C_{2} / k^{\beta+1}+o\left(1 / k^{\beta+1}\right), \quad \beta>0, C_{1} \neq 0 \\
G_{ \pm}(k) \sim k^{\gamma}, \\
\left|V_{k}^{ \pm}\right| \sim 1 / k^{\delta}, \quad \delta>0 .
\end{gathered}
$$

We set $\mu=\min \{\beta+1+2 \gamma, \alpha(\beta+1)+\gamma\}$. Then $\|U(1)-A(1)\|=O\left(\epsilon^{p}\right)$ where the exponent $p$ is given by

$$
p= \begin{cases}\frac{1}{1+2 \alpha} \quad \text { if } \mu>(1+2 \alpha) \\ \frac{1}{1+2 \alpha}-\nu \forall \nu>0 \quad \text { if } \mu=(1+2 \alpha) \\ \frac{\beta}{(\beta+1)(1+2 \alpha)-\mu} \quad \text { if } \mu<(1+2 \alpha)\end{cases}
$$

provided that $\delta$ satisfy the following constraints: $\beta+1 \leqslant \delta \leqslant \beta+\max \{1, \mu /(1+2 \alpha)\}$.

Remark: Let us mention that it can be shown that in case $\alpha=\beta=\gamma=1$ and $\delta=2$, we can take $p=1 / 3$, instead of $p=1 / 3-\nu$, for all $\nu>0$. Now, if in Lemma 2.1, the right member were missing the terms $\epsilon\left|u_{0}-t\right| / g_{t}^{2}+\epsilon\left|u_{1}-s\right| / g_{s}^{2}$, as one would expect with a little more information on the spectrum, an analysis similar to the one provided above leads to an error term of order $\epsilon^{1 / 3}$. This makes it reasonable to expect that in such a situation the error actually is of that order, as it was the case in the corresponding analysis of one crossing performed in Ref. 2, see Ref. 3. Finally, it is shown in the examples below that the values $\alpha=\beta=\gamma=1$ and $\delta=2$ are generic in some sense.

\section{EXAMPLES}

We now consider a family of models for which the situation just described takes place as the effective frequency $\varpi$ takes the value zero. We start by considering the most general model for a two level system driven by a periodic field. The model can be characterized by choosing freely the eigenvalues $\lambda_{+, m}=\lambda_{+}+m \omega$ and $\lambda_{-, k}=\lambda_{-}+k \omega$ and the corresponding eigenfunctions of the form:

$$
\psi_{+, m}(\theta)=\left(\begin{array}{c}
e^{i x(\theta)} \cos z(\theta) \\
e^{i y(\theta)} \sin z(\theta)
\end{array}\right) e^{i m \theta} \quad \text { and } \psi_{-, k}(\theta)=\left(\begin{array}{c}
-e^{-i y(\theta)} \sin z(\theta) \\
e^{-i x(\theta)} \cos z(\theta)
\end{array}\right) e^{i k \theta}
$$

in which the functions $x, y$, and $z$ are periodic modulo an integer multiple of $\theta$.

Defining the unitary matrix 


$$
\Upsilon(\theta)=\left(\begin{array}{cc}
e^{i x(\theta)} \cos z(\theta) & -e^{i y(\theta)} \sin z(\theta) \\
e^{i y(\theta)} \sin z(\theta) & e^{-i x(\theta)} \cos z(\theta)
\end{array}\right)
$$

the corresponding Floquet Hamiltonian can be written as (dropping the $\theta$ dependence in the notation)

$$
K=-i \varpi \partial-i \varpi \Upsilon\left(\partial \Upsilon^{-1}\right)+\Upsilon D \Upsilon^{-1}
$$

where $D=\operatorname{diag}\left(\lambda_{+}, \lambda_{-}\right)$. Using the notation $2 \wp=x+y, 2 \vartheta=y-x$ and choosing, without loss of generality, $\lambda_{+}=-\lambda_{-}=\lambda$, the Floquet Hamiltonian can be expressed as

$$
K=-i \varpi \partial+\left(\begin{array}{cc}
\varpi \partial \vartheta+(\lambda-\varpi \partial \wp) \cos (2 z) & (-i \varpi \partial z+(\lambda-\varpi \partial \wp) \sin (2 z)) e^{-2 i \vartheta} \\
(i \varpi \partial z+(\lambda-\varpi \partial \wp) \sin (2 z)) e^{2 i \vartheta} & -\varpi \partial \vartheta-(\lambda-\varpi \partial \wp) \cos (2 z)
\end{array}\right)
$$

where $\partial f$ denotes the derivative with respect to $\theta$. Note that when $\varpi=0$ the operator $K$ reduces to the (matrix) multiplication operator by $\Upsilon(\theta) D \Upsilon^{-1}(\theta)$ on $L^{2}\left(\mathrm{~S}^{1}, \mathrm{C}^{2}\right)$, whose spectrum consists of two eigenvalues $\pm \lambda$ which are infinitely degenerate. This is to be compared with the general situation where $K$ for $\varpi=0$ becomes a multiplication operator by an arbitrary $2 \pi$ periodic $2 \times 2$ matrix $H(\theta)$. In that case, the spectrum of $K$ is continuous and given by two band functions which are the instantaneous (in $\theta$ ) eigenvalues of $H(\theta)$.

We will consider two different models with the same eigenvalues but with different eigenfunctions. We remark that since the validity of the adiabatic theorem depends only on the properties of the eigenvalues (and regularity properties of the projectors), it gives the same upper bound for the correction for all the models (15) with equal spectrum. However, it is clear that the theorem is useful if the couplings between considered levels are nonzero. With this regard, we discuss below two examples that have the same spectrum, with an infinite number of crossings. For the first one, which is the widely used RWA (rotating wave approximation) model of quantum optics, the couplings are all equal to zero, except one (see below). The second model is a perturbation of the first one that yields nonzero couplings between the levels.

We choose, for example, the following eigenvalues:

$$
\lambda_{ \pm, k}(\varpi)=k \varpi \pm(\eta(\varpi)+\varpi) / 2, \quad \text { where } \eta(\varpi)=\sqrt{\left(\varpi-\omega_{0}\right)^{2}+\Omega^{2}}
$$

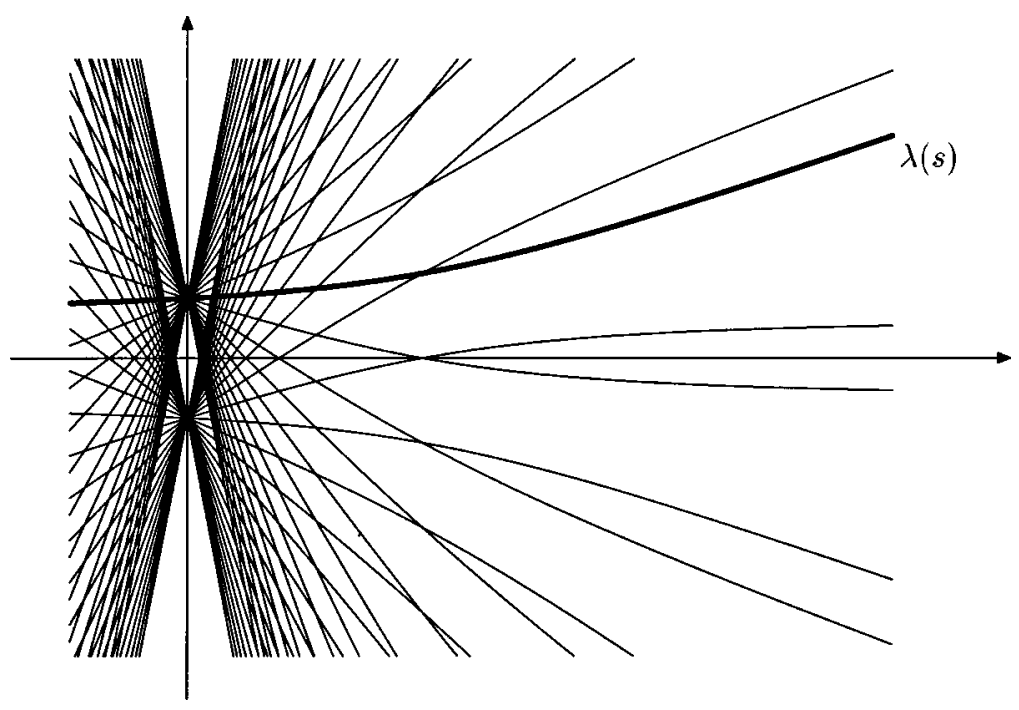

FIG. 3. The first eigenvalues of the RWA and modified RWA models. 
and $\omega_{0}, \Omega$ are constants. The first model is defined by choosing $x(\theta)=0, y(\theta)=\theta$, i.e., $2 \vartheta(\theta)$ $=2 \wp(\theta)=\theta$ and $\cos (2 z)=-\left(\varpi-\omega_{0}\right) / \eta(\varpi), \sin (2 z)=\Omega / \eta(\varpi)$, hence $z$ is independent of $\theta$. The corresponding Floquet Hamiltonian is given by

$$
K_{\mathrm{RWA}}(\theta)=-i \varpi \frac{\partial}{\partial \theta}+\frac{1}{2}\left(\begin{array}{cc}
\omega_{0} & \Omega e^{-i \theta} \\
\Omega e^{i \theta} & -\omega_{0}
\end{array}\right) .
$$

The second model is defined by the choice $x(\theta)=-\varrho(\theta) / 2, y(\theta)=\theta-\varrho(\theta) / 2$, i.e., $2 \vartheta(\theta)=\theta$, $2 \wp(\theta)=\theta-\varrho(\theta)$ and the same $z$ as for the RWA case. This leads to

$$
K_{M}(\theta)=K_{\mathrm{RWA}}(\theta)+\frac{\varpi}{2 \eta} \partial \varrho\left(\begin{array}{cc}
\omega_{0}-\varpi & \Omega e^{-i \theta} \\
\Omega e^{i \theta} & \varpi-\omega_{0}
\end{array}\right) .
$$

We consider now a supplementary smooth slow time dependence in the parameter $\varpi$ $=\varpi(s)$ and in $\varrho=\varrho(\theta, s)$. This implies that the eigenvalues, the eigenvectors and the corresponding eigenprojectors are smooth functions of $s$, so that the regularity Hypothesis $(H 0)$ (i) is satisfied. We show in appendix that (H0)(ii) is satisfied as well for any choice of smooth functions $x$, $y, z$, and $\lambda$.

We assume, for simplicity, that $\varpi(s)=s$ (but any other smooth monotonic function of $s$ would equally do). This choice corresponds to the chirping that is most often realized in experiments. We select the eigenvalue $\lambda(s)=\lambda_{+, 0}(s)=(\eta(s)+s) / 2$ and denote by $\psi$ the associated eigenvector (see Fig. 3). The only crossings that $\lambda$ experiences are with the $\lambda_{-, k+1}$ 's and they take place at times $s$ such that

$$
\eta(s)=k s, \quad k \in \mathbb{Z}^{*} .
$$

We remark however that these crossings can lead to corrections to adiabaticity, or not, depending on whether the corresponding eigenvectors are coupled. The nonadiabatic coupling among the branches is measured by the following scalar product:

$$
\begin{aligned}
\left\langle\psi(s) \mid \partial_{s} \psi_{-, k+1}(s)\right\rangle & =-\frac{1}{2 \pi} \int_{0}^{2 \pi} e^{i(k+1) \theta-2 i \wp(\theta, s)}\left(z^{\prime}(s)-i \sin (2 z(s)) \vartheta^{\prime}(\theta, s)\right) d \theta \\
& =-\frac{z^{\prime}(s)}{2 \pi} \int_{0}^{2 \pi} e^{i k \theta+i \varrho(\theta, s)} d \theta,
\end{aligned}
$$

where the ' denotes the derivative with respect to $s$.

Recall that the couplings between the eigenstate $\psi(s)$ associated with the level $\lambda(s)$ and its orthogonal complement in the Hilbert space is given by the operator $L(s)=i\left[P^{\prime}(s), P(s)\right]$, see (7), since the adiabatic evolution $A(s)$ follows the instantaneous eigenspaces. A direct computation of the matrix elements $\left\langle\psi_{-, k+1}(s) \mid L(s) \psi(s)\right\rangle$ with $P^{\prime}(s)=\left|\psi^{\prime}(s)\right\rangle\langle\psi(s)|+| \psi(s)\rangle\left\langle\psi^{\prime}(s)\right|$ shows that the above scalar product is proportional to the couplings responsible for the nonadiabatic transitions.

For the RWA model, as $\varrho=0$ the nonadiabatic couplings are given by

$$
\left\langle\psi(s) \mid \partial_{s} \psi_{-, k+1}(s)\right\rangle=-z^{\prime}(s) \delta_{k, 0} .
$$

Thus, the level $\lambda(s)$ is not coupled to the infinitely many other levels it crosses. Hence we are led in this case to an effective problem displaying no crossing, so that the error is of order $\epsilon$ in this case.

For the other model, we will obtain nonzero couplings at all the crossings, if we choose $\varrho(\theta, s)$ such that $\exp (i \varrho(\theta, s))$ has infinitely many nonzero Fourier components. For example, one can take $\varrho(\theta, s)=\rho(s) \sin (\theta)$ (in particular $\rho$ can be chosen constant). This coupling is then given by 


$$
\left\langle\psi(s) \mid \partial_{s} \psi_{-, k+1}(s)\right\rangle=(-1)^{k+1} z^{\prime}(s) J_{k}(\rho(s)),
$$

where $J_{k}$ is a Bessel function.

We will now verify that the assumptions of Proposition 3.1 are satisfied. Let us focus on the interval $(0, S]$, for $S$ small enough. The interval $[-S, 0)$ can be treated similarly. Again to simplify the notations we will not explicit the + sub/superscripts.

Remark: The preceding two examples have been chosen for their simplicity and explicit complete analytical solvability. However, we emphasize that the following analysis is valid for all the models (15) under the sole assumption that the eigenvalues can be written as $\lambda_{ \pm, m}(s)=m s$ $\pm \aleph(s) / 2$, where $\aleph$ is a $C^{2}$ function with bounded derivatives such that $\aleph(0)>0$. In particular they are satisfied for the eigenvalues given in (16). The hypotheses imply that the function $f_{\zeta}(s)$ $=\aleph(s)-\zeta s$ is strictly decreasing for any $\zeta$ greater than, say, some $\zeta_{0}$. Under these conditions the following assertion shows that the crossings that $\lambda(s)=\aleph(s) / 2$ experiences with the rest of the spectrum take place at times such that $\mathfrak{\aleph}(s)=k s, k \in \mathbb{N}$ large enough. Again, the actual corrections to adiabaticity will depend on the particular properties of the associated eigenvectors which are measured by the scalar product $\left\langle\psi(s) \mid \partial_{s} \psi_{-, k+1}(s)\right\rangle$, which generically will not be zero for an infinite number of crossings.

Assertion 1: For $\zeta \geqslant \zeta_{0}$, the function $f_{\zeta}(s)=\aleph(s)-\zeta s$ has a unique positive zero $o_{\zeta}$ and if $\zeta<\xi$ we have $o_{\zeta}>o_{\xi}$.

From the expansion

$$
f_{\zeta}(s)=\aleph(0)+\left(\aleph^{\prime}(0)-\zeta\right) s+O\left(s^{2}\right)
$$

we obtain the behavior of $o_{\zeta}$ :

$$
o_{\zeta}=\frac{\aleph(0)}{\zeta-\aleph^{\prime}(0)}+O\left(1 / \zeta^{3}\right) .
$$

We define the sequence $u_{k}>0$ by the equation:

$$
\aleph\left(u_{k}\right)-k u_{k}=(k+1) u_{k}-\aleph\left(u_{k}\right), \quad \text { i.e., } \aleph\left(u_{k}\right)=(k+1 / 2) u_{k} .
$$

Assertion 1 implies that $u_{k}<o_{k}<u_{k-1}$ and, from Eq. (18) and the fact that $u_{k}=o_{k+1 / 2}$, we obtain

$$
u_{k}=\frac{\aleph(0)}{k+1 / 2-\aleph^{\prime}(0)}+O\left(1 / k^{3}\right) \text {. }
$$

Next, we have

Assertion 2: On the interval $\left[u_{k}, u_{k-1}\right]$, the spectral gap is given by

$$
g(s)=\operatorname{dist}(\lambda(s), \sigma(s) \backslash\{\lambda(s)\})=|\aleph(s)-k s| \leqslant u_{k-1} / 2 .
$$

More precisely, for $u_{k} \leqslant s \leqslant o_{k}$ we have that $g(s)=\mathfrak{N}(s)-k s \leqslant u_{k} / 2$ and for $o_{k} \leqslant s \leqslant u_{k-1}$ we have that $g(s)=k s-\mathfrak{\aleph}(s) \leqslant u_{k-1} / 2$.

This assertion is easily proven by considering the different cases.

We now prove that the spectral hypothesis $(H 1)-(H 2)$ are verified. Assertion 1 and Equation (20) show that the sequence $\left\{u_{k}\right\}$ is (for $k$ large enough) monotonically decreasing to $a=0$. To define the intervals $V_{k}$, we choose any point $r_{k}$ in $\left(o_{k}, u_{k-1}\right)$ such that $g\left(r_{k}\right)=k r_{k}-\kappa\left(r_{k}\right)$ $\leqslant u_{k} / 2$ and set $V_{k}=\left(u_{k}, r_{k}\right)$. The $V_{k}$ 's are disjoint and $I_{k}=\left\{u_{k}\right\} \cup\left[r_{k}, u_{k-1}\right]$. By definition of $V_{k}$, we have that $g(s) \leqslant u_{k} / 2=g\left(u_{k}\right)$ and for $r_{k} \leqslant s \leqslant u_{k-1}$ the gap is given by $g(s)=k s-\aleph(s)$ $\geqslant u_{k} / 2$. Whence, hypothesis $(H 1)$ is satisfied. Finally to prove that $(H 2)$ holds, we need to estimate the behavior of $g(s)$ on $V_{k}$ : the mean value theorem implies that for each $s \in V_{k} \backslash\left\{o_{k}\right\}$, there is a $q_{s}$, in the interval joining $s$ and $o_{k}$, such that

$$
g(s)=|\aleph(s)-k s|=\left|k-\aleph^{\prime}\left(q_{s}\right)\right|\left|s-o_{k}\right| \sim k\left|s-o_{k}\right|,
$$


which shows that (H2) is satisfied with $\alpha=1$ and $G(k) \sim k$.

It remains to check the conditions given in the statement of Proposition 3.1. We have

$$
\begin{gathered}
\left|u_{k}-0\right|=u_{k}=\aleph(0) / k+\aleph(0)\left(\aleph^{\prime}(0)-1 / 2\right) / k^{2}+O\left(1 / k^{3}\right), \quad \text { i.e., } \beta=1 \\
G(k) \sim k \quad \text { i.e., } \gamma=1, \\
\left|V_{k}\right| \sim 1 / k^{2} \quad \text { i.e., } \delta=2 .
\end{gathered}
$$

To get the estimate for $\left|V_{k}\right|$, we have used that $\left(u_{k}, o_{k}\right] \subset V_{k} \subset\left(u_{k}, u_{k-1}\right]$ and the expressions for $o_{k}$, and $u_{k}$ in Eqs. (18) and (20). This implies that, $\mu=\alpha(\beta+1)+\gamma=1+2 \alpha$ and $\delta=\beta+1$. So, we can use the second case of Proposition 3.1 to prove that the adiabatic approximation holds for the models:

$$
\|U(1)-A(1)\| \leqslant c \epsilon^{p}, \quad \text { for any } p<\frac{1}{3} .
$$

In keeping with the first remark of Sec. III, we recall that a more careful analysis yields $p$ $=1 / 3$.

\section{ACKNOWLEDGMENTS}

Supports from the EC contract ERBCHRXCT94-0460 for the project "Stability and universality in classical mechanics", from the CNRS and from grants of the Fonds National Suisse de la Recherche Scientifique are acknowledged.

\section{APPENDIX A: TECHNICALITIES}

In this appendix, we show that an operator on $L^{2}\left(S^{1}, \mathcal{H}\right)$ of the form

$$
K(s, \theta)=-i \varpi(s) \frac{\partial}{\partial \theta}+H(s, \theta),
$$

where $H(s, \theta)$ is a bounded operator in $\mathcal{H}$ such that $s \mapsto H(s, \theta)$ and $s \mapsto \partial / \partial \theta H(s, \theta)$ are norm continuous and $s \mapsto \varpi(s)$ is continuous, admits a strongly continuous unitary propagator $U(s)$ $=U(s, 0)$ with all expected regularity properties, even if there is a value $a$ for which $\varpi(a)=0$. Notice that the assumptions on $H$ will be satisfied if, for example, $(s, \theta) \mapsto H(s, \theta)$ is strongly $C^{1}$.

The proof relies on a theorem of Kato, ${ }^{17}$ which we will restate in a more suitable form for our purpose.

Theorem A.1 (Kato). Let $\mathcal{K}$ and $\mathcal{D}$ be Hilbert spaces such that $\mathcal{D}$ is densely and continuously embedded in $\mathcal{K}$ and let $K(t), 0 \leqslant t \leqslant T$, be a family of self-adjoint operators in $\mathcal{K}$. Suppose that

(1) $\mathcal{D} \subset \operatorname{dom} K(t)$ for all $0 \leqslant t \leqslant T$, whence the $K(t)$ are bounded operators from $\mathcal{D}$ to $\mathcal{K}$, and the application $t \mapsto K(t)$ is norm continuous from $\mathcal{D}$ to $\mathcal{K}$;

(2) there exists a family of isomorphisms $S(t)$ from $\mathcal{D}$ to $\mathcal{K}$ which is strongly continuously differentiable and such that

$$
S(t) K(t) S(t)^{-1}=K(t)+B(t)
$$

where $B(t)$ is a strongly continuous bounded operator on $\mathcal{K}$.

Under those conditions, there exists a unique family of unitary operators $U(t, s)$ on $\mathcal{K}$ defined for $0 \leqslant s, t \leqslant T$ with the following properties:

(i) $\quad U(t, s)$ is strongly continuous on $\mathcal{K}$ in $s, t$ with $U(s, s)=\mathbb{1}$;

(ii) $U(t, r)=U(t, s) U(s, r)$;

(iii) $U(t, s) \mathcal{D} \subset \mathcal{D},\|U(t, s)\|_{\mathcal{D}} \leqslant N e^{c|t-s|}$ and is strongly continuous on $\mathcal{D}$ in $s, t$ simultaneously;

(iv) $\quad(d / d s) U(t, s) \psi=i U(t, s) K(s) \psi$ for any $\psi \in \mathcal{D}$, for $0 \leqslant s, t \leqslant T$; 
(v) for each $\psi \in \mathcal{D}$ and fixed $s,(d / d t) U(t, s) \psi$ exists and is equal to $-i K(t) U(t, s) \psi$ and strongly continuous in $\mathcal{K}$ in $t$.

To prove this theorem, we apply Theorem 6.1 in Ref. 17 to the operator $A(t)=i K(t)$, which is stable with constants of stability $c=0$ and $N=1$ (see Definition 3.1 and Theorem 4.1 therein). The fact that $U(t, s)$ is unitary follows from the self-adjointness of $K(t)$, the construction of $U(t, s)$ by unitary approximants given in the proofs of Theorem 4.1 and 6.1 in Ref. 17 and the invertibility of $U(t, s)$, which is a consequence of the fact that $A^{o}(t)=-i K(T-t)$ satifies also the hypothesis of Theorem 6.1 in Ref. 17. See also Remark 5.3 therein.

We now prove that the family of self-adjoint operators defined by Eq. (A1) satisfies the hypothesis of Theorem A.1. To simplify the notation, we will not explicit the $\theta$-dependence and write $\partial$ for $\partial / \partial \theta$.

Proof: For $\mathcal{D}$, we choose $\operatorname{dom}\left(-i w_{*} \partial\right)$ for some $w_{*}>0$, and we notice that for any $t$ such that $\varpi(t) \neq 0$, we have that dom $K(t)=\mathcal{D}$ and if $\varpi(t)=0$, then $\operatorname{dom} K(t)=\mathcal{K}$. For the norm on $\mathcal{D}$, we choose the graph norm associated to $-i w_{*} \partial$ :

$$
\|\psi\|_{\mathcal{D}}^{2}=\|\psi\|^{2}+\left\|-i w_{*} \partial \psi\right\|^{2} \geqslant\|\psi\|^{2} .
$$

Whence, $\mathcal{D}$ is a dense continuously embedded subspace of $\mathcal{K}$. For any $s, t$ and any $\psi \in \mathcal{D}$, we have

$$
\begin{aligned}
\|(K(t)-K(s)) \psi\|^{2} & \left.\leqslant 2 \frac{|\varpi(t)-\varpi(s)|^{2}}{w_{*}^{2}}\left\|-i w_{*} \partial \psi\right\|^{2}+2 \| H(t)-H(s)\right) \psi \|^{2} \\
& \leqslant 2 \max \left\{\frac{|\varpi(t)-\varpi(s)|^{2}}{w_{*}^{2}} ;\|H(t)-H(s)\| 2\right\}\|\psi\|_{\mathcal{D}}^{2} .
\end{aligned}
$$

which shows the norm continuity of $K(t)$.

We set $S(t)=S=-i w_{*} \partial+i . S$ is an isomorphism between $\mathcal{D}$ and $\mathcal{K}$ which is strongly differentiable (by $t$ independence). It remains to show that $S$ satisfies Hypothesis (2) of Theorem A.1. For this, we first notice that for any $\psi \in \operatorname{dom} K(t)$, we have that $S^{-1} \psi \in \mathcal{D} \subset \operatorname{dom} K(t)$ and

$$
\begin{aligned}
K(t) S^{-1} \psi=S^{-1} K(t) \psi+H(t) S^{-1} \psi-S^{-1} H(t) \psi & =S^{-1} K(t) \psi+S^{-1} S H(t) S^{-1} \psi-S^{-1} H(t) \psi \\
& =S^{-1}\left(K(t)-i w_{*} \partial H(t) S^{-1}\right) \psi
\end{aligned}
$$

Whence, for any $\psi \in \operatorname{dom} K(t)$, we have that the left-hand side of Eq. (A2) belongs to $\mathcal{D}$. So we can write,

$$
S K(t) S^{-1} \psi=K(t) \psi-i w_{*} \partial H(t) S^{-1} \psi, \text { for all } \psi \epsilon \operatorname{dom} K(t)
$$

Setting $B(t)=-i w_{*} \partial H(t) S^{-1}$, we have a strongly continuous bounded operator (by the assumptions on $H$ ) which satisfies $S K(t) S^{-1} \supset K(t)+B(t)$. To show the reverse inclusion, we can consider any $b \geqslant 2 \sup _{t}\|B(t)\|$ which implies that $i b$ belongs to the resolvent set of both $K(t)$ $+B(t)$ and $S K(t) S^{-1}$. It follows that $(K(t)+B(t)+i b)^{-1} \subset S(K(t)+i b)^{-1} S^{-1}$. But since the left hand side has domain $\mathcal{K}$, we must have equality between $K(t)+B(t)$ and $S K(t) S^{-1}$ instead of inclusion.

In the examples of Sec. IV, both $H(s, \theta)$ defined through (15) by means of smooth functions $x, y, z, \lambda$ of $(s, \theta)$, and $H(s, \theta)+\epsilon i\left[P^{\prime}(s, \theta), P(s, \theta)\right]$ where $P(s, \theta)=|\psi(s, \theta)\rangle\langle\psi(s, \theta)|$ with $\psi(s, \theta)$ given by one of the vectors (14) satisfy the hypotheses of the theorem. Hence assumption $(H 0)$ (ii) is satisfied for these models. 


\section{APPENDIX B: PROOF OF LEMMA 2.1, THEOREM 2.1, AND PROPOSITION 3.1}

Proof of Lemma 2.1: The idea of the proof is to integrate Eq. (7) over the interval $\left[u_{0}, u_{1}\right]$ and then to get "nice" estimates of the sizes on each subintervals $\left[u_{0}, t\right],[t, s]$, and $\left[s, u_{1}\right]$. By integrating Eq. (7), we get

$$
\begin{aligned}
i\left(W\left(u_{1}\right)-W\left(u_{0}\right)\right)= & -\int_{u_{0}}^{t} A^{-1}(u) L(u) A(u) W(u) d u-\int_{t}^{s} A^{-1}(u) L(u) A(u) W(u) d u \\
& -\int_{s}^{u_{1}} A^{-1}(u) L(u) A(u) W(u) d u .
\end{aligned}
$$

For the middle term, we simply use the properties of the operator norm and the fact that $A(u)$ and $W(u)$ are unitary to obtain

$$
\|W(s)-W(t)\| \leqslant \int_{t}^{s}\|L(u)\| d u \leqslant \sup _{u \in[0,1]}\|L(u)\||s-t|,
$$

i.e., we do not care about the behavior of $g(u)$ inside the subinterval $[t, s]$. To estimate the first integral, let $Q(u)=1-P(u)$. A simple computation, using $P(s) P^{\prime}(s) P(s) \equiv 0$, shows that

$$
P(u) L(u) P(u)=Q(u) L(u) Q(u)=0,
$$

and due to the intertwining property of $A(u)$, we can write

$$
W(t)-W\left(u_{0}\right)=i \int_{u_{0}}^{t}\left(P(0) A^{-1}(u) L(u) A(u) Q(0)+Q(0) A^{-1}(u) L(u) A(u) P(0)\right) W(u) d u .
$$

Now, we need to extract an explicit $\epsilon$ dependence from this equality in order to obtain the estimates stated in the lemma. To do this, we follow Ref. 11 and introduce the bounded operator $\mathcal{R}_{L}(u)$ defined by

$$
\mathcal{R}_{L}(u)=\frac{1}{2 i \pi} \oint_{\Gamma(u)} R(u, \lambda) L(u) R(u, \lambda) d \lambda,
$$

where $R(u, \lambda)=(H(u)-\lambda)^{-1}$ is the resolvent of $H(u)$ at $\lambda$ and where the loop $\Gamma(u)$ is a circle centered at $\lambda(u)$ of radius $g(u) / 2$. It has the properties (see Refs. 11 and 13)

$$
\begin{gathered}
{\left[\mathcal{R}_{L}(u), H(u)\right]=[L(u), P(u)],} \\
P(u) \mathcal{R}_{L}(u) P(u)=Q(u) \mathcal{R}_{L}(u) Q(u)=0 .
\end{gathered}
$$

Standard arguments show that $\mathcal{R}_{L}(u)$ is strongly $C^{1}$ and that

$$
\begin{aligned}
\mathcal{R}_{L}^{\prime}(u)= & \frac{1}{2 i \pi} \oint_{\Gamma(u)}\left(R(u, \lambda) L^{\prime}(u) R(u, \lambda)-R(u, \lambda) H^{\prime}(u) R(u, \lambda) L(u) R(u, \lambda)\right. \\
& \left.-R(u, \lambda) L(u) R(u, \lambda) H^{\prime}(u) R(u, \lambda)\right) d \lambda,
\end{aligned}
$$

where $H^{\prime}(u) R(u, \lambda)$ is to be understood as the bounded operator

$$
H^{\prime}(u) R(u, \lambda)=H^{\prime}(u) R(u, i)(\mathbb{1}+(\lambda-i) R(u, \lambda)) .
$$

Hence, we get the following estimates: 


$$
\begin{gathered}
\left\|\mathcal{R}_{L}(u)\right\| \leqslant \frac{|\Gamma(u)|}{2 \pi}\|L(u)\|(g(u) / 2)^{-2}=2\|L(u)\| / g(u), \\
\left\|\mathcal{R}_{L}^{\prime}(u)\right\| \leqslant c \max \left\{\left\|H^{\prime}(u) R(u, i)\right\|\|L(u)\|,\left\|L^{\prime}(u)\right\|\right\} / g^{2}(u) .
\end{gathered}
$$

The main property of $\mathcal{R}_{L}(u)$ (see Ref. 11) is that it satisfies for any $\psi \in \mathcal{D}$ the following equalities, as verified by means of (B5):

$$
\begin{aligned}
P(0) A^{-1}(u) L(u) A(u) Q(0) \psi= & -i \epsilon \frac{d}{d u}\left(P(0) A^{-1}(u) \mathcal{R}_{L}(u) A(u) Q(0) \psi\right) \\
& +i \epsilon P(0) A^{-1}(u) \mathcal{R}_{L}^{\prime}(u) A(u) Q(0) \psi
\end{aligned}
$$

and

$$
\begin{aligned}
Q(0) A^{-1}(u) L(u) A(u) P(0) \psi= & i \epsilon \frac{d}{d u}\left(Q(0) A^{-1}(u) \mathcal{R}_{L}(u) A(u) P(0) \psi\right) \\
& -i \epsilon Q(0) A^{-1}(u) \mathcal{R}_{L}^{\prime}(u) A(u) P(0) \psi
\end{aligned}
$$

These equations imply that $\int_{u_{0}}^{t} A^{-1}(u) L(u) A(u) W(u) d u$ is proportional to $\epsilon$. Indeed, Equalities (B3) and the intertwining property of $A(u)$ show that the diagonal blocks are 0.

Introducing Equalities (B11) and (B12) in Eq. (B4), we get

$$
\begin{aligned}
W(t)-W\left(u_{0}\right)= & -\epsilon \int_{u_{0}}^{t} \frac{d}{d u}\left(Q(0) A^{-1}(u) \mathcal{R}_{L}(u) A(u) P(0)\right. \\
& \left.-P(0) A^{-1}(u) \mathcal{R}_{L}(u) A(u) Q(0)\right) W(u) d u-\epsilon \int_{u_{0}}^{t}\left(P(0) A^{-1}(u) \mathcal{R}_{L}^{\prime}(u) A(u) Q(0)\right. \\
& \left.-Q(0) A^{-1}(u) \mathcal{R}_{L}^{\prime}(u) A(u) P(0)\right) W(u) d u .
\end{aligned}
$$

Performing an integration by part in the first integral, using the differential equation (7) for $W(u)$ and taking into account that $A(u), W(u)$ are unitary and $P(0), Q(0)$ are projectors, gives us the following bound for the norm of the difference $W(t)-W\left(u_{0}\right)$ :

$$
\begin{aligned}
\left\|W(t)-W\left(u_{0}\right)\right\| \leqslant & 2 \epsilon\left(\left\|\mathcal{R}_{L}(t)\right\|+\left\|\mathcal{R}_{L}\left(u_{0}\right)\right\|+\sup _{u \in\left[u_{0}, t\right]}\left\|\mathcal{R}_{L}(u)\right\|\|L(u)\|\left(t-u_{0}\right)\right. \\
& \left.+\sup _{u \in\left[u_{0}, t\right]}\left\|\mathcal{R}_{L}^{\prime}(u)\right\|\left(t-u_{0}\right)\right) .
\end{aligned}
$$

Next, we use first Estimates (B9) and (B10) and then the fact that $0 \leqslant u_{0}<t \leqslant 1$ to obtain the desired bound:

$$
\begin{aligned}
\left\|W(t)-W\left(u_{0}\right)\right\| \leqslant & \frac{8 \epsilon}{g_{t}} \sup _{u \in\left[u_{0}, t\right]}\|L(u)\|+\frac{4 \epsilon}{g_{t}} \sup _{u \in\left[u_{0}, t\right]}\|L(u)\|^{2}\left(t-u_{0}\right)+c \frac{2 \epsilon}{g_{t}^{2}} \\
& \times \sup _{u \in\left[u_{0}, t\right]}\left\{\left\|H^{\prime}(u) R(u, i)\right\|\|L(u)\|,\left\|L^{\prime}(u)\right\|\right\}\left(t-u_{0}\right) \\
\leqslant & 12 \frac{\epsilon}{g_{t}} \sup _{u \in[0,1]}\left\{\|L(u)\|,\|L(u)\|^{2}\right\}+2 \frac{\epsilon\left|t-u_{0}\right|}{g_{t}^{2}} \\
& \times \sup _{u \in[0,1]}\left\{\left\|H^{\prime}(u) R(u, i)\right\|\|L(u)\|,\left\|L^{\prime}(u)\right\|\right\} \\
\leqslant & c_{2}\left(\frac{\epsilon}{g_{t}}+\frac{\epsilon\left|t-u_{0}\right|}{g_{t}^{2}}\right) .
\end{aligned}
$$


Using the same kind of arguments, shows that on the subinterval $\left[s, u_{1}\right]$, we have

$$
\left\|W\left(u_{1}\right)-W(s)\right\| \leqslant c_{2}\left(\frac{\epsilon\left|s-u_{1}\right|}{g_{s}^{2}}+\frac{\epsilon}{g_{s}}\right) .
$$

Combining estimates (B2), (B15), and (B16) gives the announced bound for $\left\|W\left(u_{1}\right)-W\left(u_{0}\right)\right\|$.

Proof of Theorem 2.1: In the sequel, we will denote by the same symbol $c$ all inessential constants. Let us consider the interval $[0 ; a)$. In order to simplify the notations, we will not write the subscripts/superscripts - Picking some $t, s \in V_{k}$ such that $t<o_{k}<s$ and $\left|t-o_{k}\right|=\left|s-o_{k}\right|$, we get

$$
\begin{aligned}
\left\|W\left(u_{k}\right)-W\left(u_{k-1}\right)\right\| & \leqslant c\left(\epsilon\left|t-u_{k-1}\right| / g_{t}^{2}+\epsilon\left|s-u_{k}\right| / g_{s}^{2}+\epsilon / g_{t}+\epsilon / g_{s}+|t-s|\right) \\
& \leqslant c\left(\epsilon \frac{\Delta(k)}{G(k)^{2}}\left|t-o_{k}\right|^{-2 \alpha}+\epsilon \frac{1}{G(k)}\left|t-o_{k}\right|^{-\alpha}+\left|t-o_{k}\right|\right) \\
& \leqslant c\left(\epsilon \frac{\Delta(k)}{G(k)^{2}}\left|t-o_{k}\right|^{-2 \alpha}+\epsilon \frac{\Delta^{\alpha}(k)}{G(k)}\left|t-o_{k}\right|^{-2 \alpha}+\left|t-o_{k}\right|\right) \\
& \leqslant c\left(\epsilon \tau(k)\left|t-o_{k}\right|^{-2 \alpha}+\left|t-o_{k}\right|\right)
\end{aligned}
$$

by the preceding section. Indeed, we have that $g_{t}=\inf _{u \in\left[u_{k-1}, t\right]} g(u)=g\left(r_{t}\right)$ for some $r_{t}$ $\in\left[u_{k-1}, t\right]$. Now, by Hypothesis $(H 1), r_{t} \in V_{k}$. Whence, we have that

$$
g_{t}=g\left(r_{t}\right) \geqslant G(k)\left|r_{t}-o_{k}\right|^{\alpha} \geqslant G(k)\left|t-o_{k}\right|^{\alpha}
$$

as $r_{t} \leqslant t \leqslant o_{k}$. Using the same kind of arguments, we can show that $g_{s}=\inf _{u \in\left[s, u_{k}\right]} g(u)$ $\geqslant G(k)\left|s-o_{k}\right|^{\alpha}$. Finally to obtain the bound (B17), it remains to notice that $|s-t|=\left|t-o_{k}\right|+\mid s$ $-o_{k}|=2| t-o_{k} \mid$ together with $\left|t-o_{k}\right|,\left|t-u_{k-1}\right| \leqslant \Delta(k)$ and $\left|s-o_{k}\right|,\left|s-u_{k}\right| \leqslant \Delta(k)$.

We now get an estimate by choosing $t=t(\epsilon, k)$ in order to balance the two contributions appearing in the last term of Eq. (B17) above: for some constant $\varsigma>0$, we set

$$
\frac{\varsigma^{1+2 \alpha} \epsilon \tau(k)}{\left|t(\epsilon, k)-o_{k}\right|^{2 \alpha}}=\left|t(\epsilon, k)-o_{k}\right|,
$$

i.e.,

$$
\left|t(\epsilon, k)-o_{k}\right|=\varsigma(\epsilon \tau(k))^{1 /(1+2 \alpha)} .
$$

By definition, $t(\epsilon, k) \in V_{k}$, hence, as $k$ will eventually be bounded from above by $K(\epsilon)$, this imposes Condition (13) in the statement of the theorem. Replacing $t$ by $t(\epsilon, k)$ in (B17) and summing over $k$, we get for any $K \leqslant K(\epsilon)$,

$$
\left\|W(0)-W\left(u_{K}\right)\right\| \leqslant c\left(s+s^{-2 \alpha}\right) \sum_{k=1}^{K}(\epsilon \tau(k))^{1 /(1+2 \alpha)} .
$$

On the other hand, using the differential Eq. (7), we obtain

$$
\left\|W\left(u_{K}\right)-W(a)\right\| \leqslant \int_{u_{K}}^{a}\|L(u)\| d u \leqslant c\left|u_{K}-a\right| .
$$

Again, we balance the two right-hand sides in (B21) and (B22) by setting the integer $K=K(\epsilon)$, which has been defined in Eq. (12). Consequently, 


$$
\begin{aligned}
\|W(0)-W(a)\| & \leqslant c\left(\left(\mathrm{~s}+\mathrm{s}^{-2 \alpha}\right) \epsilon^{1 /(1+2 \alpha)} \sum_{k=1}^{K(\epsilon)} \tau(k)^{1 /(1+2 \alpha)}+\left|u_{K(\epsilon)}-a\right|\right) \\
& \leqslant c\left(\mathrm{~s}+\mathrm{s}^{-2 \alpha}+1\right)\left|u_{K(\epsilon)}-a\right| \equiv C(\mathrm{~s})\left|u_{K(\epsilon)}-a\right|,
\end{aligned}
$$

where $C(\mathrm{~s})$ is independent of $\epsilon$. Proceeding similarly on $(a, 1]$ completes the proof.

Remark: In the step (B17) we deliberately lost a little in the estimate by using $\left|t-o_{k}\right|^{-\alpha}$ $\leqslant \Delta_{k}^{\alpha}\left|t-o_{k}\right|^{-2 \alpha}$ in order to simplify the subsequent arguments. It is nevertheless possible to get slightly sharper results by not adopting this simplification, however the analysis gets more involved and less transparent. We simply note here that in the examples discussed in this paper, this more careful analysis yields, for the generic situation, an error term of order $\epsilon^{p}$ with an exponent $p=1 / 3$, instead of the value $p=1 / 3-\nu$, for any $\nu>0$ obtained there.

Proof of Proposition 3.1: The idea of the proof is to explicit conditions on the different exponents ensuring the validity of Theorem 2.1. We will only consider the interval $[0, a)$, the same kind of arguments will apply on $(a, 1]$. Again, in order to simplify the notations we will let aside the subscripts/superscripts - .

First, we have that $2 \Delta(k)=u_{k}-u_{k-1}=C_{1} \beta / k^{\beta+1}+o\left(1 / k^{\beta+1}\right) \sim 1 / k^{\beta+1}$, which implies that

$$
\delta \geqslant \beta+1>0,
$$

since $2 \Delta(k) \geqslant\left|V_{k}\right| \sim 1 / k^{\delta}$. Notice that the length of the $V_{k}$ can be rescaled by a uniform constant if $\delta=\beta+1$.

Next, $\Delta(k) / G^{2}(k) \sim 1 / k^{\beta+1+2 \gamma}$ and $\Delta^{\alpha}(k) / G(k) \sim 1 / k^{\alpha(\beta+1)+\gamma}$. So, if we denote by $\mu=\min \{\beta+1+2 \gamma, \alpha(\beta+1)+\gamma\}$ then $\tau(k)=\max \left\{\Delta(k) / G^{2}(k), \Delta^{\alpha}(k) / G(k)\right\} \sim 1 / k^{\mu}$ by increasing the overall constant in Theorem 2.1 if necessary. Whence,

$$
\sum_{k=1}^{K} \tau(k)^{1 /(1+2 \alpha)} \sim \sum_{k=1}^{K} k^{-\mu /(1+2 \alpha)} \sim\left\{\begin{array}{l}
K^{0} \text { if } \mu>1+2 \alpha \\
\log K \quad \text { if } \mu=1+2 \alpha \\
K^{1-\mu /(1+2 \alpha)} \text { if } \mu<1+2 \alpha
\end{array}\right.
$$

and considering the definition of $K(\epsilon)$ [see Eq. (12)], we obtain

$$
\epsilon^{1 /(1+2 \alpha)} \sim \frac{\left|u_{K(\epsilon)}-a\right|}{\sum_{k=1}^{K(\epsilon)} \tau(k)^{1 /(1+2 \alpha)}} \sim\left\{\begin{array}{l}
K(\epsilon)^{-\beta} \quad \text { if } \mu>1+2 \alpha \\
K(\epsilon)^{-\beta} / \log K(\epsilon) \quad \text { if } \mu=1+2 \alpha \\
K(\epsilon)^{-\beta-1+\mu /(1+2 \alpha)} \text { if } \mu<1+2 \alpha
\end{array} .\right.
$$

Condition (13) stated in Theorem 2.1 reads

$$
\varsigma(\epsilon \tau(k))^{1 /(1+2 \alpha)} \leqslant\left|V_{k}\right| / 2
$$

for all $1 \leqslant k \leqslant K(\epsilon)$. Notice that this condition is automatically satisfied if $\delta<\mu /(1+2 \alpha)$. In general, it will be satisfied for a sufficiently small $s$, if

$$
F(\epsilon) \equiv \epsilon^{1 /(1+2 \alpha)} K(\epsilon)^{\delta-\mu /(1+2 \alpha)}
$$

remains bounded as $\epsilon \rightarrow 0$. Using (B26), we have

$$
F(\epsilon) \sim\left\{\begin{array}{l}
K(\epsilon)^{\delta-\beta-\mu /(1+2 \alpha)} \quad \text { if } \mu>1+2 \alpha, \\
K(\epsilon)^{\delta-1-\beta} / \log K(\epsilon) \quad \text { if } \mu=1+2 \alpha, \\
K(\epsilon)^{\delta-\beta-1} \quad \text { if } \mu<1+2 \alpha .
\end{array}\right.
$$

As $K(\epsilon) \rightarrow \infty$ for $\epsilon \rightarrow 0$, Eq. (B29) implies that $F(\epsilon)$ will remain bounded if $\delta \leqslant \beta+$ $\max \{1 ; \mu /(1+2 \alpha)\}$. 
Hence, using (B23) and (B26), we get that the adiabatic theorem (2.1) holds with a remainder term on $[0, a)$,

$$
O\left(\left|u_{K_{-}(\epsilon)}-\alpha\right|\right)=O\left(K(\epsilon)^{-\beta}\right)=O\left(\epsilon^{p}\right)
$$

where the exponent $p$ is given by

$$
p= \begin{cases}\frac{1}{1+2 \alpha} \quad \text { if } \mu>(1+2 \alpha) \\ \frac{1}{1+2 \alpha}-\nu \forall \nu>0 \quad \text { if } \mu=(1+2 \alpha) \\ \frac{\beta}{(\beta+1)(1+2 \alpha)-\mu} \quad \text { if } \mu<(1+2 \alpha)\end{cases}
$$

provided that $\beta+1 \leqslant \delta \leqslant \beta+\max \{1 ; \mu /(1+2 \alpha)\}$. To determine $p$ in case $\mu=1+2 \alpha$ and $\delta=\beta+1$, we have used the estimate $\epsilon^{-1 /(1+2 \alpha)} \sim K(\epsilon)^{\beta} \log K(\epsilon)<K(\epsilon)^{\beta+\nu^{\prime}}$ for all $\nu^{\prime}>0$. This ends the proof of the proposition.

${ }^{1}$ S. Guérin, F. Monti, J.-M. Dupont, and H. R. Jauslin, "On the Relation Between Cavity-Dressed States, Floquet States, RWA and Semiclassical Models,' J. Phys. A 30, 7193-7215 (1997).

${ }^{2}$ M. Born and V. Fock, “Beweis des Adiabatensatzes," Z. Phys. 51, 165-180 (1928).

${ }^{3}$ G. Hagedorn, “'Adiabatic Expansions near Eigenvalue Crossings,” Ann. Phys. (N.Y.) 196, 278-295 (1989).

${ }^{4}$ S. Guérin, "Complete dissociation by chirped laser pulses designed by adiabatic Floquet analysis," Phys. Rev. A 56, $1458-1462$ (1997)

${ }^{5}$ S. Chelkowski, A. D. Bandrauk, and P. B. Corkum, "Efficient molecular dissociation by a chirped ultrashort infrared laser pulse,’’ Phys. Rev. Lett. 65, 2355-2358 (1990).

${ }^{6}$ J. E. Avron and A. Elgart "Adiabatic Theorem without a Gap Condition," Commun. Math. Phys. 203, 445-463 (1999).

${ }^{7}$ J. E. Avron and A. Elgart, "An Adiabatic Theorem without a Gap Condition," to appear in Proceedings of Qmath7, Prague (1998).

${ }^{8}$ A. Joye and Ch.-Ed. Pfister, "Exponential Estimates in Adiabatic Quantum Evolution,"' to appear in: Proceedings of the XIIth International Congress of Mathematical Physics, Brisbane, 13-19 July 1997.

${ }^{9}$ T. Kato, "On the Adiabatic Theorem of Quantum Mechanics," J. Phys. Soc. Jpn. 5, 435-439 (1950).

${ }^{10}$ G. Nenciu, "On the Adiabatic Theorem of Quantum Mechanics," J. Phys. A 13, L15-L18 (1980).

${ }^{11}$ J. E. Avron, R. Seiler, and L. G. Yaffe, “Adiabatic Theorems and Applications to the Quantum Hall Effect," Commun. Math. Phys. 110, 33-49 (1987).

${ }^{12}$ J. Avron, J. Howland, and B. Simon, “Adiabatic Theorems for Dense Point Spectra," Commun. Math. Phys. 128, 497-507 (1990).

${ }^{13}$ A. Joye, "An Adiabatic Theorem for Singularly Perturbed Hamiltonian," Ann. Inst. Henri Poincaré, Sect. A 63 , 231-250 (1995).

${ }^{14}$ S. G. Krein, Linear Differential Equations in Banach Space (American Mathematical Society, Providence, 1971).

${ }^{15}$ M. Holthaus, "Pulse-Shape-controlled Tunnelling in a Laser Field," Phys. Rev. Lett. 69, 1596 (1992).

${ }^{16} \mathrm{~S}$. Guérin and H. R. Jauslin, "Laser-Enhanced Tunnelling Through Resonant Intermediate Levels," Phys. Rev. A 55 $1262-1275$ (1997).

${ }^{17}$ T. Kato, “Linear Evolution Equation of “Hyperbolic" Type,' J. Fac. Sci., Univ. Tokyo, Sect. 1 17, $241-258$ (1970).

${ }^{18}$ F. Bornemann, "Homogenization in time of singularly perturbed mechanical systems," Lec. Notes Math. 1687 Springer (1998). 